\title{
24. Fortbildungsseminar der Arbeitsgemeinschaft Physik und Technik
}

Arbeitsgemeinschaft Physik und Technik

Am 18. und 19. Juni 2021 soll das 24. Fortbildungsseminar der Arbeitsgemeinschaft Physik und Technik (APT) als Hybridveranstaltung in Augsburg unter der wissenschaftlichen Leitung von Dipl. Phys. Gebhard Östreicher stattfinden. Unter Einhaltung der geltenden Verordnungen und der Hygienevorschriften möchte die APT so einem Kreis von Teilnehmer*innen die Möglichkeit bieten, das Semi- nar als Präsenzveranstaltung zu besuchen. Zusätzlich bietet die AG das Seminar als Onlineveranstaltung (Live-Stream) an, um allen Interessierten die Teilnahme zu ermöglichen.

Sollte die Durchführung als Hybridveranstaltung nicht möglich sein, wird das Seminar auf eine reine Onlineveranstaltung umgestellt. Die Entscheidung des endgültigen Formats wird Dipl. Phys. Gebhard Östreicher zusammen mit dem Vorstand der APT im Laufe des Mais treffen.

Das Programm sowie die Anmeldung sind im DRG-Veranstaltungskalender zu finden.
24. Fortbildungsseminar der Arbeitsgemeinschaft Physik und Technik Augsburg

WANN? 18. und 19. Juni 2021

WO? Annahof Augsburg/Augustana Saal und online

MEHR INFORMATIONEN:

www.drg.de > Veranstaltungen > Veranstaltungskalender $>18$. Juni 24. Fortbildungsseminar der Arbeitsgemeinschaft Physik und Technik (APT) - HYBRIDVERANSTALTUNG 